\title{
Organelle DNA Synthesis before Cell Nuclear Replication is Essential for Subsequent Cell Propagation
}

\author{
Takeshi Suzuki*, Atsushi Sakai, Shigeyuki Kawano \\ and Tsuneyoshi Kuroiwa \\ Department of Plant Sciences, Graduate School of Science, \\ University of Tokyo, Hongo, Tokyo, 113 Japan
}

Accepted May 9, 1996

To preserve the genetic composition of self-replicating organelles, such as mitochondria and plastids, their respective DNAs must replicate in step with cell division, which suggests that some complex control mechanism for the synthesis and distribution of organelle DNAs is presented throughout cell division. Although molecular biological studies of mitochondrial DNA (mtDNA) and plastid DNA (ptDNA) of higher plants have made remarkable progress, the mechanism which controls the replication and distribution of organelle DNAs has not yet been clarified.

DNAs of both mitochondria and plastids are associated with specific proteins to from compact structures in situ (Kuroiwa 1982, 1991). These compactly organized DNA-protein complexes are referred to as mitochondiral nuclei (nucleoids) and plastid nuclei (nucleoids). These organelle nuclei can be made visible by staining with 4',6-diamidino-2-phenylindole (DAPI) under an epi-fluorescence microscope. Studies using DAPI-fluorescence microscopy, microautoradiography with $\left[{ }^{3} \mathrm{H}\right]$ thymidine and immunofluorescence microscopy with 5bromodeoxyuridine (BrdU) labeling of the root apex of Pelargonium zonale, Arabidopsis thaliana, Nicotiana tabacum and Oryza sativa have revealed that organelle DNA synthesis is not synchronized with cell division (Kuroiwa et al. 1992, Fujie et al. 1993a, b, Suzuki et al. 1992, 1995). Organelle DNAs are synthesized prior to cell nuclear DNA before sequential cell divisions in the root tips of these plants. However, it is difficult to analyze the physiological significance and molecular mechanisms of the preferential multiplication of these organelle DNAs in higher plants in vivo. Thus, cultured cells provide a superior alternative for such analyses. Our previous studies using molecular biological techniques and microscopic observations in the cultured tobacco cell line BY-2 showed that preferential mitochondrial and plastid DNA synthesis occurred before cell nuclear DNA synthesis after the medium was renewed (Yasuda et al. 1988, Suzuki et al. 1992). The organelle DNA levels in cells increased several-fold before the first cell division. These observations in vivo root meristems and in vitro cultured cells suggest that preferential organelle DNA synthesis before cell nuclear DNA synthesis is important for plant cell propagation.

In this paper, using BY-2 cells as a model of cell multiplication, the synthesis of organelle DNAs and cell nuclear DNA was selectively inhibited by nalidixic acid (an inhibitor of prokaryotic DNA gyrase) and aphidicolin (an inhibitor of DNA polymerase $\alpha$ ), respectively, and the correlation between intra-cellular organelle DNA levels and subsequent cell propagation was examined. The results indicate that preferential synthesis and the consequent elevated levels of organelle DNA are essential for subsequent cell propagation.

\footnotetext{
* Present address, address for correspondence: Department of Cell Biology, Institute for Molecular and Cellular Regulation, Gunma University, Maebashi, Gunma 371, Japan.
} 
Materials and methods

\section{Cultured tobacco cell BY-2}

Tobacco suspension cultured cell line BY-2, derived from Nicotiana tabacum L. cv. Bright Yellow 2, was cultured by shaking on a gyratory shaker at $130 \mathrm{rpm}$ at $26^{\circ} \mathrm{C}$ in the dark as previously described (Yasuda et al. 1988). To maintain the cultured cell line, $2 \mathrm{ml}$ of stationary phase cells were transferred weekly into $95 \mathrm{ml}$ of modified Linsmaier and Skoog's medium (LSD medium; Nagata et al. 1981). For sampling and observation of cultured cells, $2 \mathrm{ml}$ of completely stationary phase cells ( 8 to 9-day-old cells) were transferred into $95 \mathrm{ml}$ of fresh LSD medium. Growth of the BY-2 cells was monitored by counting the number of cells per ml under a microscope.

\section{Preferential inhibition of organelle or cell nuclear DNA synthesis}

To inhibit organelle DNA synthesis, nalidixic acid, which inhibits the A subunit of prokaryotic DNA gyrase, was added to the culture at a final concentration of $20 \mathrm{mg} / \mathrm{l}$. To inhibit cell nuclear DNA synthesis, aphidicolin which inhibits DNA polymerase $\alpha$ of eukaryotic cells, was added to the culture at a final concentration of $5 \mathrm{mg} / 1$.

\section{Microscopic observation of organelle and organelle nucleus}

The cell walls of cultured cells were removed enzymatically by treatment with Pectolyase Y23 and Cellulase YC (both from Seishin Pharmaceutical Co., Tokyo) as described previously (Nemoto et al. 1988). Released protoplasts were fixed and stained in a drop of $1 \%$ glutaraldehyde and $1 \mathrm{mg} / \mathrm{ml}$ of DAPI in TAN buffer [17\% (w/v) sucrose, $20 \mathrm{mM}$ Tris- $\mathrm{HCl} \mathrm{pH} 7.65,0.5$ mM EDTA, $1.2 \mathrm{mM}$ spermidine, $7 \mathrm{mM}$ 2-mercaptoethanol, $0.4 \mathrm{mM}$ PMSF]. The fixed protoplasts were squashed by applying slight pressure to the coverglass. The samples were then observed under an epi-fluorescence microscope equipped with a phase-contrast objective (BHSRFK, Olympus Optical Co., Tokyo). Photographs were taken at a magnification of $\times 330$ on $35 \mathrm{~mm}$ Fuji Neopan 400 film (Fuji Photo Film Co., Tokyo).

\section{Quantitative analysis of organelle DNA by slot-blot hybridization}

To isolate whole DNAs, equal volume of cultured tobacco cells were homogenized to a fine powder while being cooled with liquid nitrogen. After the addition of 5-volume of extraction buffer (300 mM NaCl, $50 \mathrm{mM}$ Tris- $\mathrm{HCl} \mathrm{pH} 8.0,100 \mathrm{mM}$ EDTA, 4\% SDS, 2\% Sarkosyl), samples were homogenized and boiled for $20 \mathrm{~min}$. After phenol:chloroform extraction, water-soluble fraction was recovered and whole DNAs were precipitated by centrifugation after mixing with the same volume of isopropanol. To examine the relative amount of the organelle DNAs in a cell, the whole DNAs were standardized to contain precisely equal volumes of total nuclear DNA by a series of preliminary hybridization experiments as described previously (Suzuki et al. 1992) and blotted and then hybridized with two probes of plastid and mitochondria. To examine the changes in the absolute amount of DNA from each of the organelles and cell nuclei, whole DNAs from the unit colume $(0.5 \mathrm{ml})$ of the culture were blotted. Slot-blotting onto nylon membrane filters and hybridizations were performed by standard methods. RNAs were degraded during alkali fixation of DNAs onto filters by treatment with $0.4 \mathrm{~N} \mathrm{NaOH}$ for $20 \mathrm{~min}$. A probe of cloned nuclear DNA fragment, containing rice nuclear genes for both 26 S and $18 \mathrm{~S}$ rRNA (Sano and Sano 1990), was used to detect cell nuclear DNA. A cloned fragment containing PsbA gene of tobacco ptDNA was used to detect ptDNA. A 2.0-kb cloned fragment containing the entire coding region of the rice cox I gene was used to detect mtDNA (Kadowaki et al. 1989). Probes were labeled with $\left[\alpha{ }^{32} \mathrm{P}\right] \mathrm{dCTP}$ using a DNA labeling kit under the conditions recommended by the manufacturer (Amersham 
International PLC, Amersham). The intensity of hybridization signals was measured by a densitometer (Densitogragh AE-6900, ATTO Co., Tokyo).

\section{Results}

\section{Selective inhibition of DNA synthesis in the organelles and the cell nucleus}

To examine the effect of intra-cellular levels of organelle DNA on cell propagation, we used two specific inhibitors, organelle DNA synthesis and cell nuclear DNA synthesis were inhibited by nalidixic acid and aphidicolin, respectively. We first examined the effects of these inhibitors on cell proliferation and DNA synthesis in organelles and cell nuclei.

The cultured tobacco cells of BY-2 multiplied about 50-fold in a week when $2 \mathrm{ml}$ of stationary-phase cells were transferred to $95 \mathrm{ml}$ of fresh normal LSD medium (Fig. 1). Quantitative hybridization experiments exactly standardized with cell nuclear DNA amount revealed that the intra-cellular organelle DNA levels increased 2-fold within $24 \mathrm{hr}$, remained at these high levels until $72 \mathrm{hr}$, and decreased thereafter (Fig. 2). Cell multiplication was completely inhibited in medium containing $20 \mathrm{mg} / 1 \mathrm{nalidixic}$ acid. The intra-cellular levels of mitochondria and plastid DNA decreased to 50\% within $24 \mathrm{hr}$, and remained at these low levels for 2 days (Fig. 2). Since the fluorescence intensities of the organelle nucleoids did not change until the third day (Fig. 3), this decrease is not because of the degradation of the organelle DNA but relative decrease by the increase of the cell nuclear DNAs. The effect of nalidixic acid on organelle DNA synthesis was examined by quantitative hybridization experiment standardized with the unit volume of the culture (Fig. 4). When cells were cultured in the medium containing $20 \mathrm{mg} / 1$ nalidixic acid, both mitochondria and plastids DNAs were not increased. This result shows that nalidixic acid inhibits the organelle DNA synthesis. Since the nalidixic acid was added to the low organelle DNA level cells just after the transfer, the cells were not propagated, and the increase of cell nuclear DNA was not observed (Fig. 4a). The results shown in Fig. 2 b suggest that the cell nuclear DNA relatively increases below 2-fold. In this experiment (Fig. 4), however, since the total DNA amounts of each of the organelles and cell nuclei in an unit volume of the culture $(0.5 \mathrm{ml})$ were measured by quantitative Southern blot analysis, the detection of such small change bellw 2-fold was very difficult. Moreover, the experiments of Figs. 5 and 6 showed that the cells which had a high level of organelle DNA were able to propagate even in the nalidixic acid containing medium. These results strongly

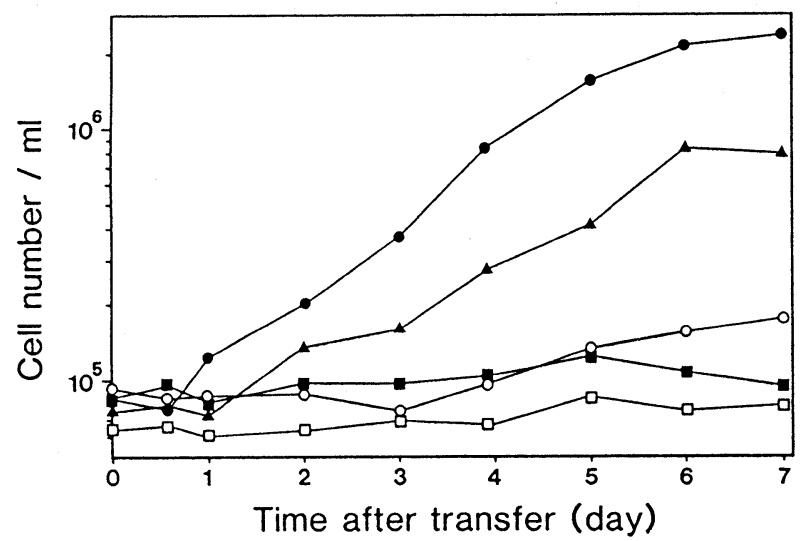

Fig. 1. Effect of specific inhibitors of DNA synthesis on BY-2 cell multiplication. Growth curves are expressed as cell number per $1 \mathrm{ml}$ of culture with normal LSD medium (๑), or medium containing $2 \mathrm{mg} / 1$ of nalidixic acid $(\mathbf{\Delta}), 20 \mathrm{mg} / 1$ of nalidixic acid $(\bigcirc), 200 \mathrm{mg} / 1$ of nalidixic acid (ם), or $5 \mathrm{mg} / 1$ of aphidicolin $(\square)$. 


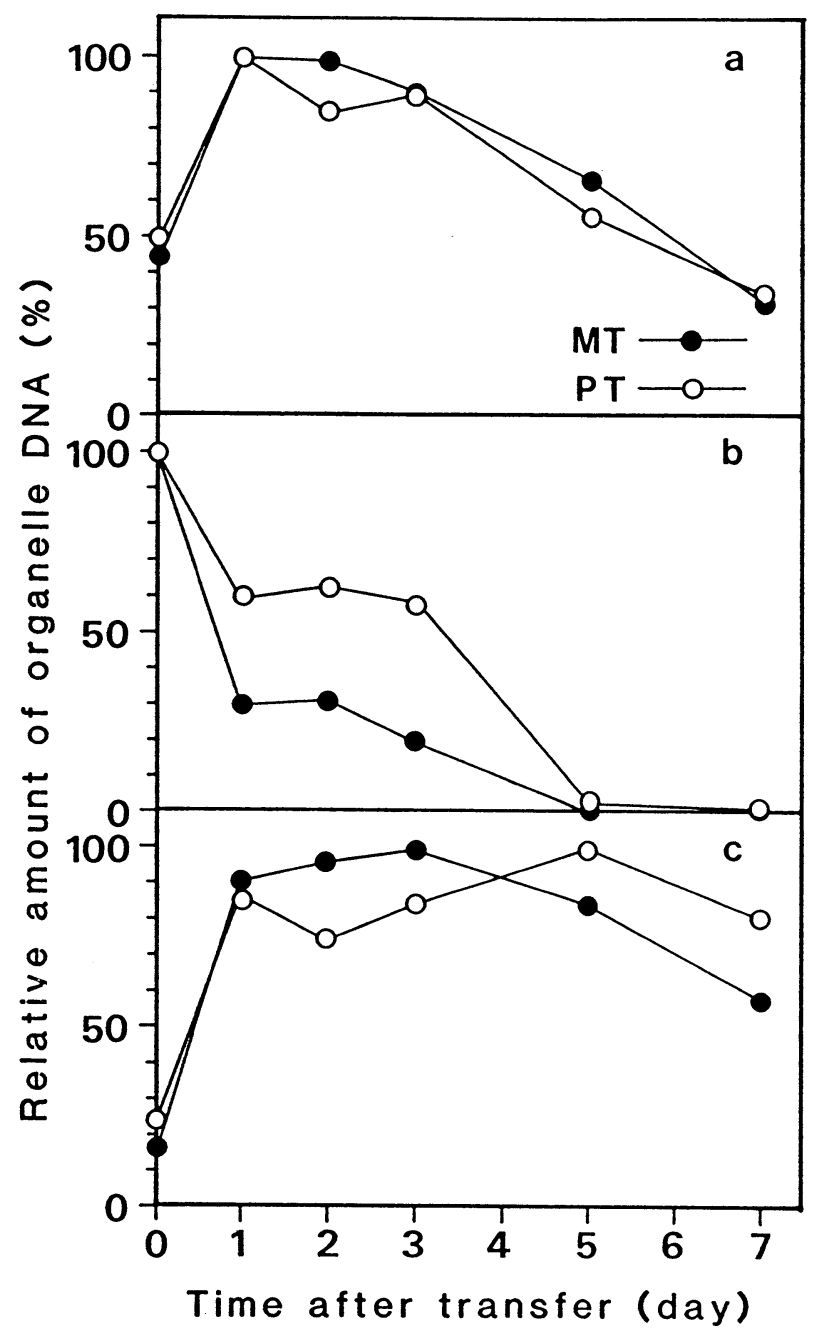

Fig. 2. Relative changes in the DNA levels of both mitochondria $(\bullet)$ and plastids $(\bigcirc)$ per cell nuclei in BY-2 during culture with normal LSD medium (a), medium containing $20 \mathrm{mg} / 1$ nalidixic acid (b), or medium containing $5 \mathrm{mg} / 1$ aphidicolin (c). Relative organelle DNA levels were determined by measuring the intensities of hybridization signals with a densitometer.

suggest that the nalidixic acid does not inhibit cell nuclear DNA synthesis directly. Cell multiplication was also completely inhibited by the addition of aphidicolin at a final concentration of $5 \mathrm{mg} / \mathrm{l}$ (Fig. 1). However, the intra-cellular DNA levels of mitochondria and plastids increased about 5.8- and 3.7-fold, respectively, within $24 \mathrm{hr}$, and remained high throughout the culture (Fig. 2). This result shows that aphidicolin specifically inhibited cell nuclear DNA synthesis, and did not inhibit organelle DNA synthesis.

\section{Morphological changes of organelle nuclei during culture with inhibitors}

We examined the effect of nalidixic acid and aphidicolin on morphologies of organelles and their nuclei by DAPI fluorescence microscopy (Fig. 3). At the time of transfer, spherical plastids and elongated mitochondria were observed, but their fluorescence intensities were quite weak. When the cells were transferred to control medium, the fluorescence of organelle nuclei intensified within $24 \mathrm{hr}$ after the transfer. During this period, the plastids became extremely elongated and the mitochondria became small and granular. After the third day, the 

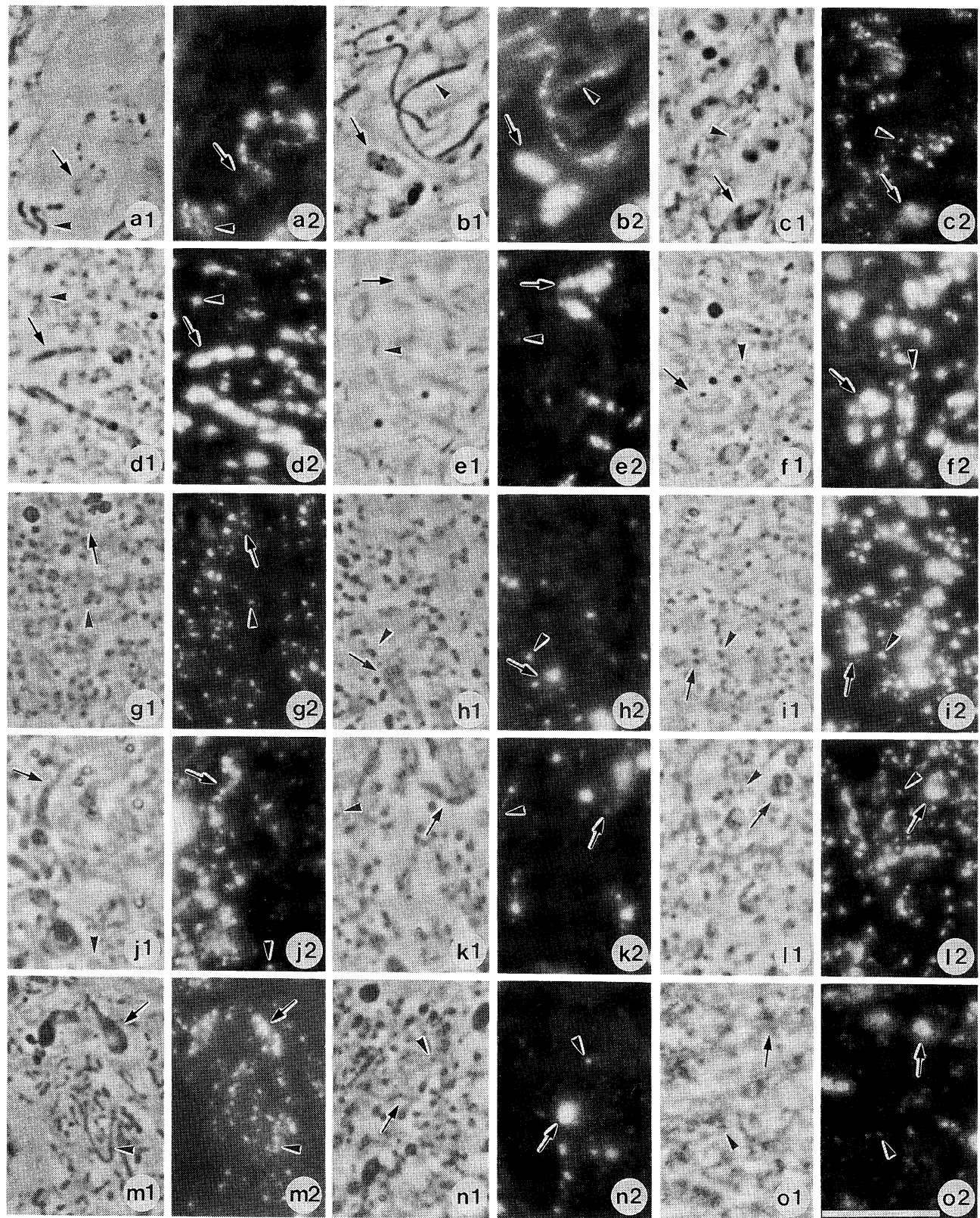

Fig. 3. Phase-contrast $(\mathrm{a} 1-\mathrm{o} 1)$ and epi-fluorescence (a2-o2) images of organelle and organelle nuclei of BY-2 during culture with specific inhibitors. Cells were transferred to normal LSD medium (a, d, g, j, m), medium containing $20 \mathrm{mg} / 1$ nalidixic acid (b, e, h, k, n), or medium containing $5 \mathrm{mg} / \mathrm{l}$ aphidicolin (c, f, i, 1, o). Cells harvested at 0 (a, b, c), 24 (d, e, f), 72 (g, h, i), $120(\mathrm{j}, \mathrm{k}, \mathrm{l})$, and $168(\mathrm{~m}, \mathrm{n}, \mathrm{o}) \mathrm{hr}$ after the transfer were treated with cellulolytic enzymes to revome cell walls, and were observed under an epi-fluorescence microscope equipped with a phase-contrast objective after staining with DAPI. Small arrowheads and large arrowheads indicate a mitochondrion, a plastid and their nuclei, respectively. Bar, $10 \mu \mathrm{m}$.

fluorescence intensity of organelle nuclei decreased gradually. Elongated plastids divided into small pieces after the third day, and mitochondria gradually elongated after the fifth day. In contrast, when cells were grown in medium containing $20 \mathrm{mg} / 1$ of nalidixic acid, the fluorescence of organelle nuclei did not intensify, and the shapes of the organelles remained 


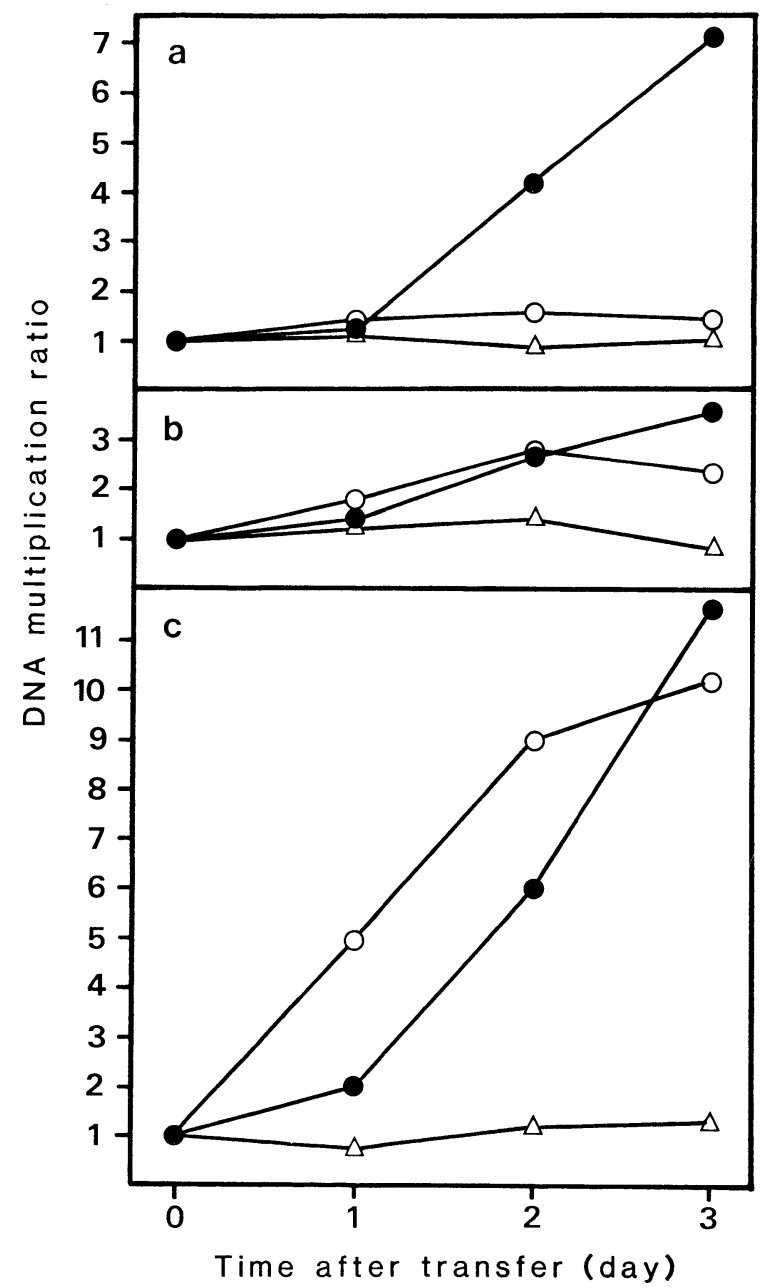

Fig. 4. Changes in the DNA levels of cell nuclei (a), mitochondria (b), and plastids (c) per equal volume of the culture with normal medium (•), medium containing $20 \mathrm{mg} / 1$ of nalidixic acid $(\triangle)$, and $5 \mathrm{mg} / \mathrm{ml}$ of aphidicolin $(\bigcirc)$. DNA levels were determined by measuring the intensities of hybridization signals with a densitometer.

essentially the same as those at the time of transfer until the third day. After the third day, the fluorescence intensity of organelle nuclei decreased gradually. Since the morphology of the cells gradually became abnormal after the third day and at the seventh day more than $50 \%$ of the cells were abnormal, this decrease may be occurred by the organelle DNA degradation in dying cells. In the culture with aphidicolin $(5 \mathrm{mg} / \mathrm{l})$, the fluorescence intensities of organelle nuclei increased several-fold within $24 \mathrm{hr}$, and the mitochondria assumed a granular shape. When the cells were cultured for more than $72 \mathrm{hr}$ in medium containing aphidicolin, some additional changes in cytological morphology were observed, i.e., elongated and spindle-shaped cell nuclei. We limited the treatment with aphidicolin to less than $48 \mathrm{hr}$ in the following experiments so that the effect of aphidicolin on cell nuclear cytological morphology would be negligible.

\section{Effect of intra-cellular DNA levels of organelles on the growth of the culture}

To determine the relationship between intra-cellular organelle DNA levels and cell propagation, nalidixic acid $(20 \mathrm{mg} / \mathrm{l})$ was added at various time points $(0,6,12,24,48,72 \mathrm{hr}$ after the transfer) to increase intra-cellular organelle DNA levels (Fig. 2a). The effect of 
nalidixic acid on cell multiplication was reduced as the organelle DNA levels increased, and no inhibition was observed beyond $72 \mathrm{hr}$ after the transfer (Fig. 5). This result suggests that the increase in intra-cellular organelle DNA levels was necessary for cell multiplication. However, there is a possibility that the increase in the number of cells, rather than the increase in organelle DNA levels, may have resulted in this reduced inhibition. To exclude this possibility, the effect of elevated intra-cellular organelle DNA levels on the capacity for cell multiplication was examined more quantitatively. Cells were precultured in aphidicolin-containing medium for various lengths of time $(0,6,12,48 \mathrm{hr})$ to prepare cells with various organelle DNA levels (Table 1). After the aphidicolin was washed away with fresh LSD medium, approximately equal numbers (from $3.9 \times 10^{4}$ to $5.9 \times 10^{4}$ ) of cells were transferred into medium containing nalidixic acid, and cell multiplication was then monitored. When cells with low organelle DNA levels that had not been treated with aphidicolin were transferred into medium containing nalidixic acid, cell multiplication was completely inhibited (Fig. 6a). When cells that had been treated with aphidicolin for $6 \mathrm{hr}$, in which mt- and pt-DNA levels were 2.2 and 1.7, respectively, were transferred into medium containing nalidixic acid, the cells multiplied 1.4-fold (Fig. 6b, Table 1). The cell multiplication ratio increased from 5.3- to 16.3-fold when the duration of treatment with aphidicolin was prolonged from $12 \mathrm{hr}$ to $48 \mathrm{hr}$. Along with this increase, mtDNA levels and ptDNA levels increased from 3.4 to 6.2 and from 2.4 to 3.2 , respectively (Fig. 6c, d). Cell growth curves of the control (Fig. 6a) and of aphidicolin-treated cells that had been transferred to normal LSD medium (Fig. 6b, c, d) were almost the same, indicating that aphidicolin pre-treatment has very little effect on cell multiplication. As summarized in Table 1, the mathematical correlation between intra-cellular organelle DNA levels and the cell

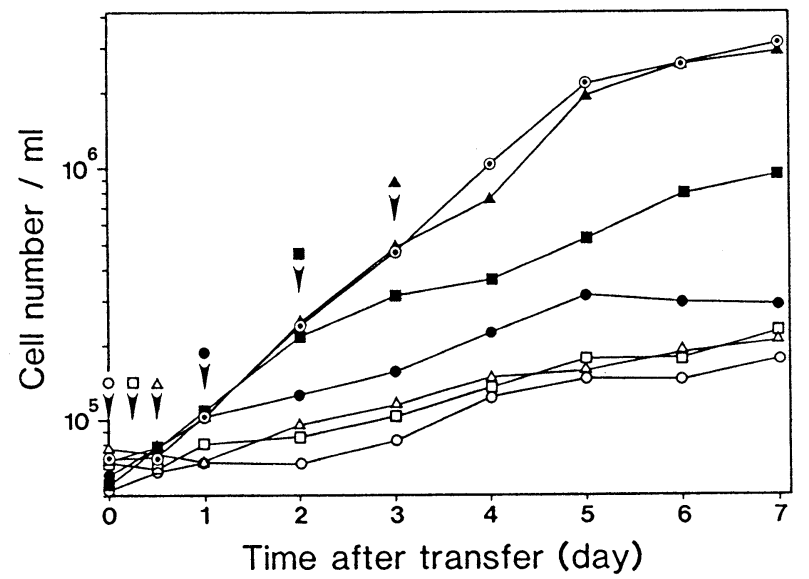

Fig. 5. Effect of the timing of the addition of nalidixic acid on cell multiplication. $2 \mathrm{mg}$ of nalidixic acid was added to $100 \mathrm{ml}$ of culture (final concentration of $200 \mathrm{mg} / \mathrm{l}$ ) at $0(\bigcirc), 6(\square)$, $12(\triangle), 24(\bullet), 48(\boldsymbol{\square})$, or $72(\boldsymbol{\Delta}) \mathrm{hr}$ after cell transfer. The line with the double circles $(\bullet)$ shows the growth curve in normal LSD medium without the inhibitor.

Table 1. Multiplication ratio of the effect of intra-cellular organele DNA levels during the culture with aphidicolin, and cell multiplication ratio that follows

\begin{tabular}{lcccc}
\hline \hline & \multicolumn{4}{c}{ Time after transfer (hr) } \\
\cline { 2 - 5 } & 0 & 6 & 12 & 48 \\
\hline Plastid DNA & 1.0 & 1.7 & 2.4 & 3.2 \\
Mitochondrial DNA & 1.0 & 2.2 & 3.4 & 6.2 \\
\hline Cell multiplication ratio & 1.2 & 1.4 & 5.3 & 16.3 \\
\hline
\end{tabular}




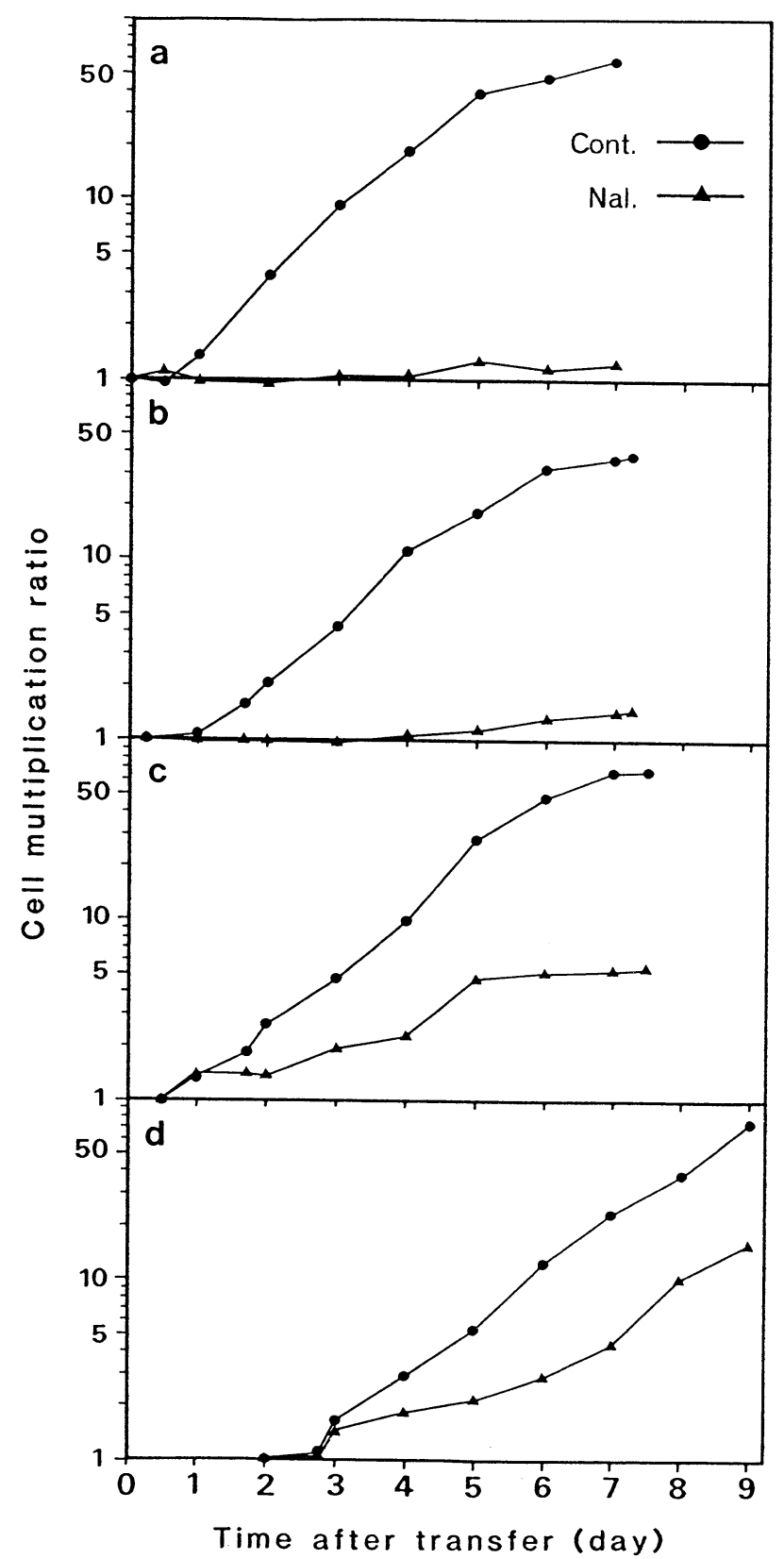

Fig. 6. Effect of intra-cellular organelle DNA levels after the inhibition of organelle DNA synthesis by nalidixic acid on cell multiplication. The cells were treated with aphidicolin for either 0 (a), 6 (b), 12 (c), or 48 (d) hr to produce cells with various levels of organelle DNA (Table 1). These cells were transferred to either normal LSD $(\bullet)$ or medium containing $20 \mathrm{mg} / 1$ nalidixic acid (A). Growth curves are presented as the cell multiplication ratio versus the number of cells transferred.

multiplication ratio was not observed, but it might be because the inhibitory effect of the nalidixic acid was weaker as the number of the cells increased. The cells whose organelle DNA levels had not increased could not proliferate in the medium in which the organelle DNA synthesis was inhibited by nalidixic acid synthesis. In contrast, when organelle DNA levels had been increased several-fold in advance, the cells proliferated even in the medium which 
contained nalidixic acid according to their organelle DNA levels. This result suggests that the increase in intra-cellular organelle DNA levels is essential for cell propagation.

\section{Discussion}

Elevation of intra-cellular mtDNA levels is commonly observed in the meristematic cells of apical meristems of the root and shoot of higher plants, as well as in cultured cells. This elevation is due to preferential synthesis of mtDNA before cell multiplication (Kuroiwa et al. 1992, Suzuki et al. 1992, 1995, Fujie et al. 1993a, b, 1994). The mtDNA levels of undifferentiated prototypic cells are several times greater than those of differentiated cells. For example, extremely giant mitochondrial nuclei, which contain as much DNA as cell nuclei, can be observed in the mature egg cells of $P$. zonale (Kuroiwa and Kuroiwa 1992). These facts suggest that the increase in intra-cellular mtDNA levels may be necessary for the preparation of mtDNAs, which are then distributed by subsequent sequential cell divisions.

In the present study, to clarify whether or not preferential synthesis of organelle DNAs is essential for plant cell propagation, we examined the relationship between intra-cellular organelle DNA levels and multiplication of cultured tobacco cell BY-2 by using two specific inhibitors, nalidixic acid and aphidicolin. Nalidixic acid, a quinolone, is known as a specific inhibitor for bacterial topoisomerase-type II (DNA gyrase). In the mammalian cells, it has been reported that nalidixic acid inhibits specifically and reversibly the mitochondrial DNA synthesis (Castra et al. 1983, Gallagher et al. 1986, Lawrence et al. 1993). In higher plants, however, there is no report of the effect specific for organelle DNA synthesis. Alternatively, it has been reported that there is a topoisomerase-type I activity which is ATP-independent and not inhibited by nalidixic acid in the higher plant mitochondrial extracts (Meissner et al. 1992). In those reports, however, they only examined the activity for super coiled DNA (pBR322) conversion to open circular form but did not examine the effect on mitochondrial DNA synthesis directly. Moreover, novobiocin, a specific inhibitor for B subunit of type II topoisomerase, inhibited the topoisomerase activity in higher plant mitochondrial lysate (Meissner et al. 1992). From our results of specific inhibition of nalidixic acid for the organelle DNA synthesis, topoisomerase-type II will act in the higher plant organelles. Inhibition of organelle DNA synthesis by nalidixic acid resulted in the termination of cell multiplication, indicating that the synthesis of organelle DNAs is a prerequisite for cell multiplication. Furthermore, a correlation was observed between intra-cellular organelle DNA levels and the capacity of the cells for proliferation; the higher the intra-cellular organelle DNA levels, the more the cells proliferated in the absence of the synthesis of organelle DNAs (Fig. 6, Table 1). This observation indicates that the reduced intra-cellular organelle DNA levels may have limited the capacity of cell proliferation.

The increase in mitochondrial DNA might be necessary to produce a high respiratory activity, since the mitochondrial DNA levels in cardiac muscle cells are reportedly 5-fold greater than those in white skeletal muscle cells in rabbit (Williams 1986). These previous findings imply that the transcriptional activity of organelles is template-limited. Apparently, intra-cellular organelle DNA levels increase when there is a clear need for rapid protein synthesis in organelles (Bendich 1987). However, there is some evidence that the relationship between transcriptional activity and DNA copy number in organelles is not particularly strong. For example, plastid DNA copy number increases less than two-fold (from 130 to 210 per plastid) while transcription increases 10-fold (Baumgartner et al. 1989). Therefore, the increase in the copy number of organelle DNAs may, at least in part, stimulate transcription. However, it apparently plays only a limited role in the activation of organelle gene expression in general. 
As we reported previously, DNAs of both mitochondria and plastids increase in meristematic cells, such as those in the root apex and heterotrophically growing cultured cells (Suzuki et al. 1992). In these meristematic cells, a high respiratory activity of mitochondria may be required to supply energy for active cells divisions, whereas plastids whose principle function is photosynthesis are relatively unnecessary. In the purpose of the elevation in organelle DNA levels is to activate organelle gene expression, then we might expect that only mitochondrial DNA is synthesized preferentially in these cells. Nevertheless, DNAs of non-green plastids are also synthesized and stored in large amounts in these meristematic cells. This indicates that the elevation of organelle DNAs in meristematic cells has some physiological significance other than the stimulation of the expression of organelle genes.

The mathematical correlation between intra-cellular organelle DNA levels and the cell multiplication ratio have not been observed, but it may be because of that the inhibitory effect of the nalidixic acid is weaken as the number of the cells increased. The cells whose organelle DNA levels had not increased could not proliferate in the medium in which the organelle DNA synthesis was inhibited by nalidixic acid synthesis. In contrast, when organelle DNA levels had been increased several-fold in advance, the cells proliferated even in the medium which contained nalidixic acid according to their organelle DNA levels. This result suggests that the increase in intra-cellular organelle DNA levels is essential for cell propagation and the intra-cellular organelle DNA levels limit the capacity for cell multiplication. Therefore, preferential organelle DNA synthesis and the resulting increase in the intra-cellular organelle DNA levels may be a mechanism for maintaining the integrity of genetic information in cells during the subsequent distribution of organelle DNAs due to cell multiplication. This may be one of the reasons why organelle DNA synthesis, and the consequent rapid increase in organelle DNA levels, are essential for the multiplication of plant cells.

\section{Summary}

Elevation of intra-cellular organelle DNA levels due to preferential synthesis of organelle DNAs before cell multiplication is generally observed in meristematic cells of higher plants, such as those in apical meristems and cultured cells. We analyzed the physiological significance of this phenomena using cultured tobacco cell BY-2 as a model system for cell multiplication in plants. Cultured tobacco cell BY-2 multiplied approximately 50-fold in a week in normal culture medium. However, when nalidixic acid, which inhibits prokaryotic DNA gyrase, was added to the culture, synthesis of organelle DNA was selectively inhibited and cell multiplication was arrested. This indicates that the synthesis of organelle DNAs is a prerequisite for cell multiplication. To quantitatively clarify the effect of the elevation of organelle DNA levels on the capacity for cell proliferation, cells with various organelle DNA levels were prepared by preculturing cells for various lengths of time in culture medium which contained aphidicolin, a specific inhibitor of eukaryotic DNA polymerase $\alpha$, and then transferring them to culture medium which contained nalidixic acid. Their growth rates in the absence of further organelle DNA synthesis were monitored. A correlation was observed between intra-cellular organelle DNA levels and the capacity of the cells to proliferate, indicating that intra-cellular organelle DNA levels limited the capacity for cell proliferation.

\section{Acknowledgements}

This work was supported by grants (to T. K., \#05269205 for Special Research on Priority Areas and to T. S., \#3189) from the Ministry of Education, Science and Culture of Japan, and by a grant for Pioneering Research Project in Biotechnology from the Ministry of Agriculture, 
Forestry and Fisheries of Japan.

\section{References}

Baumgartner, B. J., Rapp, J. C. and Mullet, J. E. 1989. Plastid transcription activity and DNA copy number increase early in barley choloroplast development. Plant Physiol. 89: 1011-1018.

Bendich, A. J. 1987. Why do chloroplasts and mitochondria contain so many copies of their genome? BioEssays 6: 279-282.

Castora, F. J., Vissering, F. F. and Simpson, M. V. 1983. The effect of bacterial DNA gyrase inhibitors on DNA synthesis in mammalian mitochondria. Biochem. Biophys. Acta. 740: 417-427.

Fujie, M., Kuroiwa, H., Kawano, S. and Kuroiwa, T. 1993a. Studies on the behavior of organelles and their nucleoids in the root apical meristem of Arabidopsis thaliana (L.) Col. Planta 189: 443-452.

-, -, Suzuki, T., Kawano S. and Kuroiwa, T. 1993b. Organelle DNA synthesis in the quiescent center of Arbidopsis thaliana (Col.). J. Exp. Bot. 44: 689-693.

-, -, Kawano, S., Mutoh, s. and Kuroiwa, T. 1994. Behavior of organelles and their nucleoids in the shoot apical meristem during leaf development in Arabidopsis thaliana L. Planta 194: 395-405.

Gallagher, M., Weinberg, R. and Simpson, M. V. 1986. Effect of the bacterial DNA gyrase inhibitors, novobiocin, nalidixic acid, and oxolinic acid, on oxidative phosphorylation. J. Biol. Chem. 261: 8604-8607.

Kadowaki, K., Suzuki, T., Kazama, S., Oh-fuchi, T. and Sakamoto, W. 1989. Nucleotide sequence of the cytochrome oxidase subunit I gene from rice mitochondria. Nucleic acids Res. 17: 7519.

Kuroiwa, H. and Kuroiwa, T. 1992. Giant mitochondria in the mature egg cell of Pelargonium zonale. Protoplasma 168: $184-188$.

Kuroiwa, T. 1982. Mitochondrial nuclei. Int. Rev. Cytol. 75: 1-59.

- 1991. The replication, differentiation, and inheritance of plastids with emphasis on the concept of organelle nuclei. Int. Rev. Cytol. 128: 1-62.

-, Fujie, M. and Kuroiwa, H. 1992. Studies on the behavior of mitochondrial DNA: Synthesis of mitochondrial DNA occurs actively in a specific region just above the quiescent center in the root meristem of Pelargonium zonale. J. Cell Sci. 101: 483-493.

Lawrence, J. W., Darkin-Rattray, S., Xi, F., Neims, A. H. and Rowe, T. C. 1993. 4-Quinolones cause a selective loss of mitochondrial DNA from mouse L1210 leukemia cells. J. Cell Biochem. 51: 165-174.

Meissner, K., Dorfel, P. and Borner, T. 1992. Topoisomerase activity in mitochondrial lysates of a higher plant (Chenopodium album L.). Biochem. Int. 27: 1119-1125.

Nagata, T., Okada, K., Takebe, I. and Matsui, C. 1981. Delivery of tobacco mosaic virus RNA into plant protoplasts mediated by reversephase evaporation vesicles (liposomes). Mol. Gen. Genet. 184: 161-165.

Nemoto, Y., Kawano, S., Nakamura, S., Mita, T., Nagata, T. and Kuroiwa, T. 1988. Studies on plastid-nuclei (nucleoids) in Nicotiana tabacum L. I. Isolation of proplastid-nuclei from cultured cells and identification of proplastid-nuclear proteins. Plant Cell Physiol. 29: 167-177.

Sano, Y. and Sano, R. 1990. Variation of the intergenic spacer region of ribosomal DNA in cultivated and wild rice species. Genome 33: 290-218.

Suzuki, T., Kawano, S., Sakai, S., Fujie, M., Kuroiwa, H., Nakamura, H. and Kuroiwa, T. 1992. Preferential mitochondrial and plastid DNA synthesis before multiple cell divisions in Nicotiana tabacum. J. Cell Sci. 103: 831-837.

-, Sasaki, N. Sakai, A., Kawano, S. and Kuroiwa, T. 1995. Localization of organelle DNA synthesis within the root apical meristem of rice. J. Exp. Bot. 46: 19-25.

Williams, R. S. 1986. Mitochondrial gene expression in mammalian striated muscle. Evidence that variation in gene dosage is the major regulatory event. J. Biol. Chem. 261: 12390-12394.

Yasuda, T., Kuroiwa, T. and Nagata, T. 1988. Preferential synthesis of plastid DNA and increased replication of plastids in cultured tobacco cells following medium renewal. Planta 174: 235-241. 